\title{
Quasiclassical approach to the spin Hall effect in the two-dimensional electron gas
}

\author{
Roberto Raimondi and Cosimo Gorini \\ Dipartimento di Fisica “E. Amaldi,” Università di Roma Tre, Via della Vasca Navale 84, 00146 Roma, Italy \\ Peter Schwab and Michael Dzierzawa \\ Institut für Physik, Universität Augsburg, 86135 Augsburg, Germany \\ (Received 23 January 2006; revised manuscript received 27 April 2006; published 27 July 2006)
}

\begin{abstract}
We study the spin-charge coupled transport in a two-dimensional electron system using the method of quasiclassical ( $\xi$-integrated) Green's functions. In particular we derive the Eilenberger equation in the presence of a generic spin-orbit field. The method allows us to study spin and charge transport from ballistic to diffusive regimes and continuity equations for spin and charge are automatically incorporated. In the clean limit we establish the connection between the spin Hall conductivity and the Berry phase in momentum space. For finite systems we solve the Eilenberger equation numerically for the special case of the Rashba spin-orbit coupling and a two-terminal geometry. In particular, we calculate explicitly the spin Hall induced spin polarization in the corners, predicted by Mishchenko et al. [Phys. Rev. Lett. 93, 226602 (2004)]. Furthermore we find universal spin currents in the short-time dynamics after switching on the voltage across the sample, and calculate the corresponding spin Hall polarization at the edges. Where available, we find perfect agreement with analytical results.
\end{abstract}

DOI: 10.1103/PhysRevB.74.035340

PACS number(s): 72.25.-b

\section{INTRODUCTION}

In the presence of spin-orbit coupling spin currents and spin polarization can be generated as a response to electric fields. ${ }^{1-5}$ Recently the spin Hall effect, i.e., a spin current that flows perpendicular to an applied electric field has been observed experimentally in electron doped semiconductors and in a two-dimensional hole system. ${ }^{6,7}$ Theoretically one may distinguish the extrinsic from the intrinsic spin Hall effect, depending on whether spin-orbit coupling arises due to scattering by impurities or from the intrinsic band structure of the samples. The intrinsic spin Hall effect has been studied for holes in $p$-type semiconductors in Ref. 4 and for electrons in $n$-type semiconductors in Ref. 5 . In both cases, the striking result is the independence of the spin Hall conductivity $\sigma_{s H}$ of the strength of the spin-orbit coupling, at least when disorder effects are ignored. Sinova et al. ${ }^{5}$ found in the two-dimensional electron system with Rashba spin-orbit coupling a universal value for the spin Hall conductivity, $\sigma_{s H}=e / 8 \pi$. Soon it was realized that the universal spin Hall conductivity still exists in the presence of a Dresselhaus term, ${ }^{8,9}$ and even in the presence of a weak in-plane magnetic field. ${ }^{10}$ It was pointed out that this may be related to a Berry phase in momentum space, i.e., the winding number of the spin-orbit field when going once around the Fermi surface. Shytov et al. ${ }^{11}$ showed such a connection explicitly in the specific case where the modulus of the spin-orbit field remains constant on the Fermi surface.

Clearly it is an important question to ask how $\sigma_{s H}$ depends on disorder. For the Rashba model the effect is quite dramatic, namely an arbitrarily weak amount of disorder fully suppresses the spin Hall conductivity. ${ }^{12-18}$ Meanwhile it is understood that this surprising result is a special property of the Rashba Hamiltonian and is related to the linear-inmomentum spin-orbit field: The time derivative of the total spin is proportional to the spin current, so that in a steady state both quantities are zero. ${ }^{16,19}$ In the case of a more gen- eral spin-orbit field, a finite spin Hall effect has been reported even in the presence of disorder. ${ }^{11,18,20-23}$

With the conventional definition of the spin current, given by the anticommutator of the velocity operator and the Pauli matrices, the spin current is not conserved. Hence it is not automatically guaranteed that a current in the bulk induces a polarization at the edges of the sample. Furthermore the spin current is not directly accessible experimentally; the measurable quantity is the spin polarization instead. Therefore it is of interest to study directly the electric field induced spin density. A strategy followed by some authors is to discretize the Rashba Hamiltonian in terms of a tight-binding model which is then studied near the band edge. In this way the spin Hall induced spin accumulation in systems with linear dimensions of several tens of the Fermi wavelength have been studied. ${ }^{24,25}$ Macroscopic systems are conveniently described in terms of semiclassical kinetic equations, ${ }^{11,13,14,21-23,26}$ and in particular in terms of diffusion equations describing the coupled dynamics of spin and charge degrees of freedom. In clean systems, however, the spin relaxation length can become comparable to the elastic mean free path. In this situation the spin dynamics is not diffusive, and one must go beyond the diffusive approximation. In what follows we present a theory of the spin Hall effect in terms of quasiclassical Green's functions, which covers the full range from clean systems to the diffusive limit. The first step is the derivation of the equation of motion, the Eilenberger equation. From there we derive the continuity equation for charge and spin, and obtain explicit expressions for the current densities. For the Rashba model it follows that no spin current can flow in a time- and space-independent situation. In the clean limit we find a solution of the equation of motion which corresponds to the universal spin Hall effect. In the general case of disordered finite systems we solve the equation of motion numerically. 


\section{THE EILENBERGER EQUATION}

We start from the Hamiltonian,

$$
H=\frac{p^{2}}{2 m}+\mathbf{b} \cdot \boldsymbol{\sigma},
$$

where $\mathbf{b}$ is the internal magnetic field due to the spin-orbit coupling and $\boldsymbol{\sigma}$ is the vector of Pauli matrices. In the Rashba model, for example, $\mathbf{b}=\alpha \mathbf{p} \times \mathbf{e}_{z}$. For a spin- $\frac{1}{2}$ particle one can write the spectral decomposition of the Hamiltonian in the form

$$
H=\epsilon_{+}|+\rangle\left\langle+\left|+\epsilon_{-}\right|-\right\rangle\langle-|,
$$

where $\epsilon_{ \pm}=p^{2} / 2 m \pm|\mathbf{b}|$ are the eigenenergies corresponding to the projectors

$$
| \pm\rangle\langle \pm|=\frac{1}{2}(1 \pm \hat{\mathbf{b}} \cdot \boldsymbol{\sigma})
$$

where $\hat{\mathbf{b}}$ is the unit vector in the $\mathbf{b}$ direction. We write Green's functions in Wigner coordinates, $G=G(\mathbf{p}, \mathbf{x})$, where $\mathbf{p}$ is the Fourier transform of the relative coordinate and $\mathbf{x}$ is the center-of-mass coordinate. For the Green's functions we make the ansatz

$$
\check{G}=\left(\begin{array}{cc}
G^{R} & G^{K} \\
0 & G^{A}
\end{array}\right)=\frac{1}{2}\left\{\left(\begin{array}{cc}
G_{0}^{R} & 0 \\
0 & -G_{0}^{A}
\end{array}\right),\left(\begin{array}{cc}
\widetilde{g}^{R} & \widetilde{g}^{K} \\
0 & \widetilde{g}^{A}
\end{array}\right)\right\},
$$

where the curly brackets denote the anticommutator. $G_{0}^{R, A}$ are retarded and advanced Green's functions in the absence of external perturbations,

$$
G_{0}^{R(A)}=\frac{1}{\epsilon+\mu-p^{2} / 2 m-\mathbf{b} \cdot \boldsymbol{\sigma}-\Sigma^{R(A)}},
$$

and $\Sigma^{R(A)}$ are the retarded and advanced self-energies which will be specified below. The ansatz guarantees that in equilibrium the matrix of Green's functions with lowercase letters is

$$
\check{\widetilde{g}}=\left(\begin{array}{cc}
1 & 2 \tanh (\epsilon / 2 T) \\
0 & -1
\end{array}\right) .
$$

The main assumption for the following is that we can determine $\check{\widetilde{g}}$ such that it does not depend on the modulus of the momentum $\mathbf{p}$ but only on the direction $\hat{\mathbf{p}}$. Under this condition $\check{\widetilde{g}}$ is directly related to the $\xi$-integrated Green's function which we denote by $\check{g}$,

$$
\check{g}=\frac{i}{\pi} \int d \xi \check{G}, \quad \xi=p^{2} / 2 m-\mu .
$$

For convenience we suppressed in the equations above spin and time arguments of the Green's function, $\check{g}$ $=\check{g}_{t_{1} s_{1}, t_{2} s_{2}}(\hat{\mathbf{p}} ; \mathbf{x})$. In some cases Wigner coordinates for the time arguments are more convenient, $\check{g} \rightarrow \check{g}_{s_{1} s_{2}}(\hat{\mathbf{p}}, \boldsymbol{\epsilon} ; \mathbf{x}, t)$.

We evaluate the $\xi$ integral explicitly in the limit where $|\mathbf{b}|$ is small compared to the Fermi energy. Since the main contributions to the $\xi$ integral are from the region near zero, it is justified to expand $\mathbf{b}$ for small $\xi, \mathbf{b} \approx \mathbf{b}_{0}+\xi \partial_{\xi} \mathbf{b}_{0}$, with the final result

$$
\begin{aligned}
& \check{g} \approx \frac{1}{2}\left\{1-\partial_{\xi} \mathbf{b}_{0} \cdot \boldsymbol{\sigma}, \check{\widetilde{g}}\right\}, \\
& \check{\widetilde{g}} \approx \frac{1}{2}\left\{1+\partial_{\xi} \mathbf{b}_{0} \cdot \boldsymbol{\sigma}, \check{g}\right\} .
\end{aligned}
$$

In the equation of motion we must also evaluate integrals of a function of $\mathbf{p}$ and a Green's function. Assuming again that $|\mathbf{b}| \ll \epsilon_{F}$ we find

$$
\frac{i}{\pi} \int d \xi f(\mathbf{p}) \check{G} \approx f\left(\mathbf{p}_{+}\right) \check{g}_{+}+f\left(\mathbf{p}_{-}\right) \check{g}_{-},
$$

where $\mathbf{p}_{ \pm}$is the Fermi momentum in the \pm subband including corrections due to the internal field, $\left|\mathbf{p}_{ \pm}\right| \approx p_{F} \mp|\mathbf{b}| / v_{F}$, and

$$
\check{g}_{ \pm}=\frac{1}{2}\left\{\frac{1}{2} \pm \frac{1}{2} \hat{\mathbf{b}}_{0} \cdot \boldsymbol{\sigma}, \check{g}\right\}, \quad \check{g}=\check{g}_{+}+\check{g}_{-} .
$$

Following the conventional procedure ${ }^{27}$ we derive now the equation of motion for $\check{g}$. From the Dyson equation and after a gradient expansion the equation of motion for the Green's function $\check{G}$ reads

$$
\partial_{t} \check{G}+\frac{1}{2}\left\{\frac{\mathbf{p}}{m}+\frac{\partial}{\partial \mathbf{p}}(\mathbf{b} \cdot \boldsymbol{\sigma}), \frac{\partial}{\partial \mathbf{x}} \check{G}\right\}+i[\mathbf{b} \cdot \boldsymbol{\sigma}, \check{G}]=-i[\check{\check{\Sigma}}, \check{G}] .
$$

The Boltzmann equation or Boltzmann-type kinetic equations are obtained by either integrating (12) over energy $\epsilon$ or over $\xi$, see Refs. 27 . Reference 11 for instance follows the first route, whereas we integrate over $\xi$. Retaining terms up to first order in $|\mathbf{b}| / \epsilon_{F}$ leads to an Eilenberger equation of the form

$$
\begin{aligned}
& \sum_{\nu= \pm}\left(\partial_{t} \check{g}_{\nu}+\frac{1}{2}\left\{\frac{\mathbf{p}_{\nu}}{m}+\frac{\partial}{\partial \mathbf{p}}\left(\mathbf{b}_{\nu} \cdot \boldsymbol{\sigma}\right), \frac{\partial}{\partial \mathbf{x}} \check{g}_{\nu}\right\}+i\left[\mathbf{b}_{\nu} \cdot \boldsymbol{\sigma}, \check{g}_{\nu}\right]\right) \\
& \quad=-i[\check{\check{L}, \check{g}] .}
\end{aligned}
$$

In the entire paper we will take the self-energy as $\check{\Sigma}=$ $-i\langle\check{g}\rangle / 2 \tau$, which corresponds to $s$-wave impurity scattering in the Born approximation; $\langle\cdots\rangle$ denotes the angular average over $\hat{\mathbf{p}}$.

To check the consistency of the equation we study at first its retarded component in order to verify that $\widetilde{g}^{R}=1$ solves the generalized Eilenberger equation. From Eq. (8) we find that $g^{R}=1-\partial_{\xi}\left(\mathbf{b}_{0} \cdot \boldsymbol{\sigma}\right)$ and using (11) we arrive at

$$
g_{ \pm}^{R}=\left(1 \mp \partial_{\xi} b\right)\left(\frac{1}{2} \pm \frac{1}{2} \hat{\mathbf{b}}_{ \pm} \cdot \boldsymbol{\sigma}\right) .
$$

Apparently both the commutators on the left- and right-hand sides of the Eilenberger equation are zero, at least to first order in the small parameter $\partial_{\xi} \mathbf{b}_{0}$. Similar arguments may also be used to verify that the equilibrium Keldysh component of the Green's function, $g^{K}=\tanh (\epsilon / 2 T)\left(g^{R}-g^{A}\right)$, solves the equation of motion.

In the appendixes we demonstrate how the frequency dependent spin Hall conductivity and the equation of motion in the diffusive limit can be obtained from Eq. (13). For the Rashba model our results agree with Ref. 13. 


\section{CONTINUITY EQUATION-VANISHING SPIN HALL CURRENT}

Equipped with the Eilenberger equation it is not difficult to see that for a spin-orbit field of the Rashba or linear Dresselhaus model the spin Hall conductivity is zero. The argument is analogous to that of Ref. 16 and makes use of the continuity equation.

When taking the angular average of the Eilenberger equation (13), the term on the right-hand side vanishes and we are left with a set of continuity equations for the charge and spin components of the Green's function. With $\check{g}_{s s^{\prime}}=\check{g}_{0} \delta_{s s^{\prime}}$ $+\check{\mathbf{g}} \cdot \boldsymbol{\sigma}_{s s^{\prime}}$ the equations read

$$
\begin{gathered}
\partial_{t}\left\langle\check{g}_{0}\right\rangle+\partial_{\mathbf{x}} \cdot \check{\mathbf{J}}_{c}=0, \\
\partial_{t}\left\langle\check{g}_{x}\right\rangle+\partial_{\mathbf{x}} \cdot \check{\mathbf{J}}_{s}^{x}=2 \sum_{\nu= \pm}\left\langle\mathbf{b}_{\nu} \times \check{\mathbf{g}}_{\nu}\right\rangle_{x}, \\
\partial_{t}\left\langle\check{g}_{y}\right\rangle+\partial_{\mathbf{x}} \cdot \check{\mathbf{J}}_{s}^{y}=2 \sum_{\nu= \pm}\left\langle\mathbf{b}_{\nu} \times \check{\mathbf{g}}_{\nu}\right\rangle_{y}, \\
\partial_{t}\left\langle\check{g}_{z}\right\rangle+\partial_{\mathbf{x}} \cdot \check{\mathbf{J}}_{s}^{z}=2 \sum_{\nu= \pm}\left\langle\mathbf{b}_{\nu} \times \check{\mathbf{g}}_{\nu}\right\rangle_{z}
\end{gathered}
$$

with

$$
\check{\mathbf{J}}_{c, s}=\sum_{\nu= \pm}\left\langle\frac{1}{2}\left\{\frac{\mathbf{p}_{\nu}}{m}+\frac{\partial}{\partial \mathbf{p}}\left(\mathbf{b}_{\nu} \cdot \boldsymbol{\sigma}\right), \check{g}_{\nu}\right\}\right\rangle_{c, s} .
$$

The densities and currents are related to the Keldysh components of $\langle\check{g}\rangle$ and of $\check{\mathbf{J}}_{c, s}$ integrated over $\boldsymbol{\epsilon}$. Explicitly the particle and spin current densities are given by

$$
\begin{aligned}
\mathbf{j}_{c}(\mathbf{x}, t) & =-\pi N_{0} \int \frac{d \epsilon}{2 \pi} \mathbf{J}_{c}^{K}(\epsilon ; \mathbf{x}, t), \\
\mathbf{j}_{s}^{i}(\mathbf{x}, t) & =-\frac{1}{2} \pi N_{0} \int \frac{d \epsilon}{2 \pi} \mathbf{J}_{s}^{K i}(\epsilon ; \mathbf{x}, t)
\end{aligned}
$$

with $N_{0}=m / 2 \pi$ being the density of states of the twodimensional electron gas. In the absence of spin-orbit coupling $(\mathbf{b}=0)$ one recovers the well-known expressions

$$
\begin{aligned}
& \mathbf{j}_{c}(\mathbf{x}, t)=-\frac{1}{2} N_{0} \int d \epsilon\left\langle\mathbf{v}_{F} g_{0}^{K}\right\rangle, \\
& \mathbf{j}_{S}^{i}(\mathbf{x}, t)=-\frac{1}{4} N_{0} \int d \epsilon\left\langle\mathbf{v}_{F} g_{i}^{K}\right\rangle .
\end{aligned}
$$

In the presence of the field $\mathbf{b}$ the expressions are in general more complex. For the Rashba model, for example, the particle current is given by the lengthy expression

$$
\begin{aligned}
\mathbf{j}_{c}(\mathbf{x}, t)= & -\frac{1}{2} N_{0} \int d \epsilon\left\{v_{F}\left\langle\hat{\mathbf{p}} g_{0}^{K}\right\rangle\right. \\
& \left.+\alpha \hat{\mathbf{e}}_{z} \times\left\langle\mathbf{g}^{K}\right\rangle-\alpha\left\langle\hat{\mathbf{p}}\left[\hat{\mathbf{p}} \cdot\left(\hat{\mathbf{e}}_{z} \times \mathbf{g}^{K}\right)\right]\right\rangle\right\} .
\end{aligned}
$$

Finally let us consider the spin current with polarization in the $z$ direction for the model with both a Rashba and a
Dresselhaus term for which the spin-orbit field reads

$$
\left(\begin{array}{l}
b_{x} \\
b_{y} \\
b_{z}
\end{array}\right)=\alpha\left(\begin{array}{c}
p_{y} \\
-p_{x} \\
0
\end{array}\right)+\beta\left(\begin{array}{c}
p_{x} \\
-p_{y} \\
0
\end{array}\right) \text {. }
$$

Because the field lies in the $x-y$ plane, $\check{\mathbf{J}}_{s}^{z}$ is simply given by $\check{\mathbf{J}}_{s}^{z}=\left\langle\mathbf{v}_{F} \check{g}_{z}\right\rangle$. Besides the field is just linear in $\mathbf{p}$. As a result we find that the source term in the continuity equations (16) and (17) can be expressed in terms of the spin current,

$$
\begin{aligned}
& (16)=2\left\langle b_{y, 0} \check{g}_{z}\right\rangle=-2 m \alpha \check{J}_{s, x}^{z}-2 m \beta \check{J}_{s, y}^{z}, \\
& (17)=2\left\langle b_{x, 0} \check{g}_{z}\right\rangle=-2 m \alpha \check{J}_{s, y}^{z}-2 m \beta \check{J}_{s, x}^{z} .
\end{aligned}
$$

In a stationary situation and for a spatially homogeneous system the left-hand side of the continuity equation is zero and this implies a vanishing spin current,

$$
j_{s, x}^{z}=j_{s, y}^{z}=0 .
$$

\section{CLEAN LIMIT-UNIVERSAL SPIN HALL CURRENTS}

We consider now the Eilenberger equation in the clean limit, $\tau \rightarrow \infty$, and study the linear response to a homogeneous electric field. For a realistic system with at least weak disorder this study still gives reliable results on short time scales, $t \ll \tau$. Generally an electric field can be included in the quasiclassical equations of motion by the substitution $\partial_{\mathbf{x}} \rightarrow \partial_{\mathbf{x}}$ $-|e| \mathbf{E} \partial_{\epsilon}$. The Keldysh component of the linearized Eilenberger equation becomes

$$
\begin{aligned}
& \sum_{\nu= \pm}\left(\partial_{t} g_{\nu}^{K}-\frac{|e|}{m} \mathbf{E} \cdot \mathbf{p}_{\nu} \partial_{\epsilon} g_{\nu}^{K, \mathrm{eq}}-\frac{|e|}{2}\left\{\left(\mathbf{E} \cdot \partial_{\mathbf{p}}\right)\left(\mathbf{b}_{\nu} \cdot \boldsymbol{\sigma}\right), \partial_{\epsilon} g_{\nu}^{K, \mathrm{eq}}\right\}\right. \\
& \left.+i\left[\mathbf{b}_{\nu} \cdot \boldsymbol{\sigma}, g_{\nu}^{K}\right]\right)=0 .
\end{aligned}
$$

In the following we focus on the spin components of the equation. Explicitly we get

$$
\partial_{t} g_{x}^{K}=2 b_{y, 0} g_{z}^{K}+|e| \mathbf{E} \cdot\left(\mathbf{P} \hat{b}_{x, 0}-\mathbf{v}_{F} \partial_{\xi} b_{x, 0}+\partial_{\mathbf{p}} b_{x, 0}\right) F_{\epsilon},
$$

$$
\begin{aligned}
& \partial_{t} g_{y}^{K}=-2 b_{x, 0} g_{z}^{K}+|e| \mathbf{E} \cdot\left(\mathbf{P} \hat{b}_{y, 0}-\mathbf{v}_{F} \partial_{\xi} b_{y, 0}+\partial_{\mathbf{p}} b_{y, 0}\right) F_{\epsilon}, \\
& \partial_{t} g_{z}^{K}=2\left(b_{x, 0} g_{y}^{K}-b_{y, 0} g_{x}^{K}\right)+2\left(b_{x, 0} \partial_{\xi} b_{y, 0}-b_{y, 0} \partial_{\xi} b_{x, 0}\right) g_{0},
\end{aligned}
$$

where for the sake of brevity $\mathbf{P}=\Sigma_{\nu} \nu \mathbf{p}_{\nu} / 2 m$ and $F_{\epsilon}$ $=2 \partial_{\epsilon} \tanh (\epsilon / 2 T)$. For the $g_{z}^{K}$ component one obtains

$$
\frac{d^{2} g_{z}^{K}}{d t^{2}}+4 b_{0}^{2} g_{z}^{K}=2 F_{\epsilon}|e|\left[b_{x, 0}\left(\mathbf{E} \cdot \partial_{\mathbf{p}}\right) b_{y, 0}-b_{y, 0}\left(\mathbf{E} \cdot \partial_{\mathbf{p}}\right) b_{x, 0}\right] \text {. }
$$

Notice that only the second of the two terms involving the electric field in Eq. (29) remains in the equation for the $g_{z}^{K}$ 
component. The solution of this differential equation is the sum of an oscillating and a time-independent term. Due to the (undamped) oscillations it is clear that a stationary solution is never reached so the arguments of the preceding section leading to vanishing spin Hall current do not apply. The time-independent solution of the differential equation is related to a zero-frequency spin current given by

$$
\mathbf{j}_{s}^{z}=-\frac{|e|}{4 \pi}\left\langle\mathbf{p}_{F}\left(\mathbf{E} \cdot \partial_{\mathbf{p}}\right) \Psi\right\rangle, \quad \tan \Psi=b_{y, 0} / b_{x, 0} .
$$

Notice that the spin current does not depend on the magnitude of the field $\mathbf{b}$, but only on the variation of its direction when going around the Fermi surface. An even more explicit result is obtained when the spin Hall conductivity tensor is antisymmetric,

$$
\begin{aligned}
\sigma_{s H} & =\frac{1}{2}\left(\sigma_{s H}^{y, x}-\sigma_{s H}^{x, y}\right) \\
& =-\frac{|e|}{8 \pi}\left\langle\left(p_{F y} \partial_{p_{x}}-p_{F x} \partial_{p_{y}}\right) \Psi\right\rangle \\
& =\frac{|e|}{8 \pi} \oint \frac{d \mathbf{p}}{2 \pi} \cdot \partial_{\mathbf{p}} \Psi,
\end{aligned}
$$

i.e., the spin Hall conductivity is the universal number $|e| / 8 \pi$ times the winding number of the internal field $\mathbf{b}$ when going once around the Fermi surface.

We notice that Eq. (34) is consistent with Refs. 8 and 9 and also with Ref. 10 where the spin Hall conductivity ignoring disorder has been calculated using the Kubo formula for a Rashba-Dresselhaus system in the presence of an inplane magnetic field. Equation (37) which relates the spin Hall conductivity with a winding number, i.e., the Berry phase in momentum space, generalizes the equivalent result of Ref. 11, where it has been assumed that the modulus of $\mathbf{b}$ is constant on the Fermi surface.

\section{FINITE AND DISORDERED SYSTEMS-NUMERICAL RESULTS}

In this section we solve Eq. (13) numerically for the Rashba model. Compared to the diffusion equations, ${ }^{13,21,26}$ one advantage of our method is that we have access to length scales that are shorter than the mean free path $l=v_{F} \tau$. This is crucial in weakly disordered systems, $|\mathbf{b}|>1 / \tau$, where the characteristic length scale for the spin polarization, the spin relaxation length, is of the order of the mean free path. Furthermore, when considering time-dependent situations we can study the time evolution on time scales which are shorter than the scattering time $\tau$.

In the following we will consider a geometry as shown in Fig. 1: A rectangular strip of length $L_{x}$ and width $L_{y}$ is connected to reservoirs at $x=0$ and $x=L$. At interfaces the Eilenberger equation must be complemented with boundary conditions. ${ }^{28-30}$ The boundary condition between the strip and the reservoirs is obtained assuming that the reservoirs are made of the same material as the strip, i.e., there is no

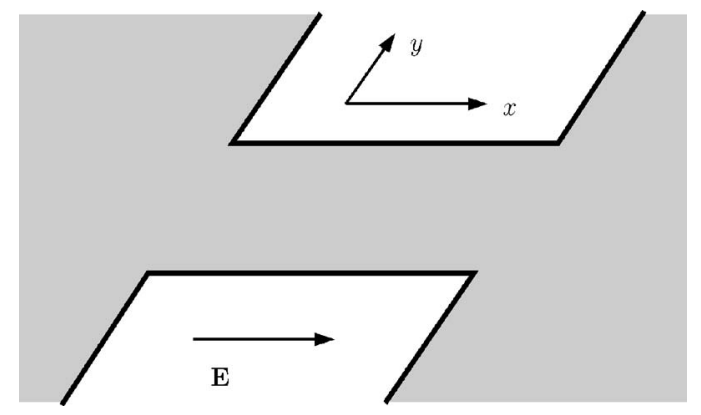

FIG. 1. The two-terminal geometry under consideration: A rectangular strip of a two-dimensional electron gas is connected to two reservoirs. We assume that the strip and the reservoirs are made of the same material, i.e., the spin-orbit field exists also in the reservoirs.

Fermi surface mismatch, and that both reservoirs are in thermal equilibrium. For directions $\hat{\mathbf{p}}$ pointing into the strip the Green's function at the interface $(x=0, L)$ reads

$$
\begin{aligned}
\left.g^{K}\left(\hat{\mathbf{p}}_{\text {in }} ; \mathbf{x}\right)\right|_{x=0, L} & =\left.g_{\text {eq }}^{K}\left(\hat{\mathbf{p}}_{\text {in }} ; \mathbf{x}\right)\right|_{\mathbf{x}} \text { in the reservoir } \\
& =\tanh \left(\frac{\boldsymbol{\epsilon} \pm|e| V / 2}{2 T}\right)\left(g^{R}-g^{A}\right),
\end{aligned}
$$

where $V$ is the applied voltage. At the boundary with an insulator the Green's functions for in- and outgoing directions are related via a surface scattering matrix. In leading order in the small parameter $\alpha / v_{F}$ the condition reads ${ }^{30}$

$$
g^{K}\left(\hat{\mathbf{p}}_{\text {out }}\right)=S g^{K}\left(\hat{\mathbf{p}}_{\text {in }}\right) S^{+} .
$$

In the absence of spin scattering at the surface for instance, the $S$ matrix equals unity in spin space and it follows that both spin and charge currents are conserved at the surface. This and related boundary conditions have been assumed in the diffusive limit in the recent literature. . $1,31,32^{2}$

Generally, the $S$ matrix can be calculated by solving the quantum mechanical surface scattering problem. We assume specular scattering and assume that boundary scattering does not induce transitions between the two spin-orbit subbands, so that

$$
\left|\mathbf{k}_{\text {in }} \pm\right\rangle \rightarrow \exp ( \pm i \vartheta)\left|\mathbf{k}_{\text {out }} \pm\right\rangle,
$$

as it is expected for smooth confining potentials. ${ }^{33}$ Explicitly the surface $S$ matrix for the Rashba Hamiltonian is

$$
S=e^{i \varphi}\left(\begin{array}{cc}
e^{i \varphi} \cos \vartheta & -\sin \vartheta \\
\sin \vartheta & e^{-i \varphi} \cos \vartheta
\end{array}\right),
$$

where the angle $\varphi$ characterizes the ingoing direction, $\hat{\mathbf{p}}_{\text {in }}$ $=(\cos \varphi, \sin \varphi)$. Our numerical results are obtained assuming that the relative phase shift in the scattering for the two subbands is negligible, i.e., $\vartheta=0$.

Finally, to integrate the equation of motion numerically we must discretize the space coordinate $\mathbf{x}$ and the Fermi surface. In dirty systems $g^{K}(\hat{\mathbf{p}})$ is nearly isotropic, so it is clear that a few discrete points $\hat{\mathbf{p}}_{i}$ on the Fermi surface are sufficient. In clean systems this is not a priori evident, but numerical tests show that even in this case convergence is 

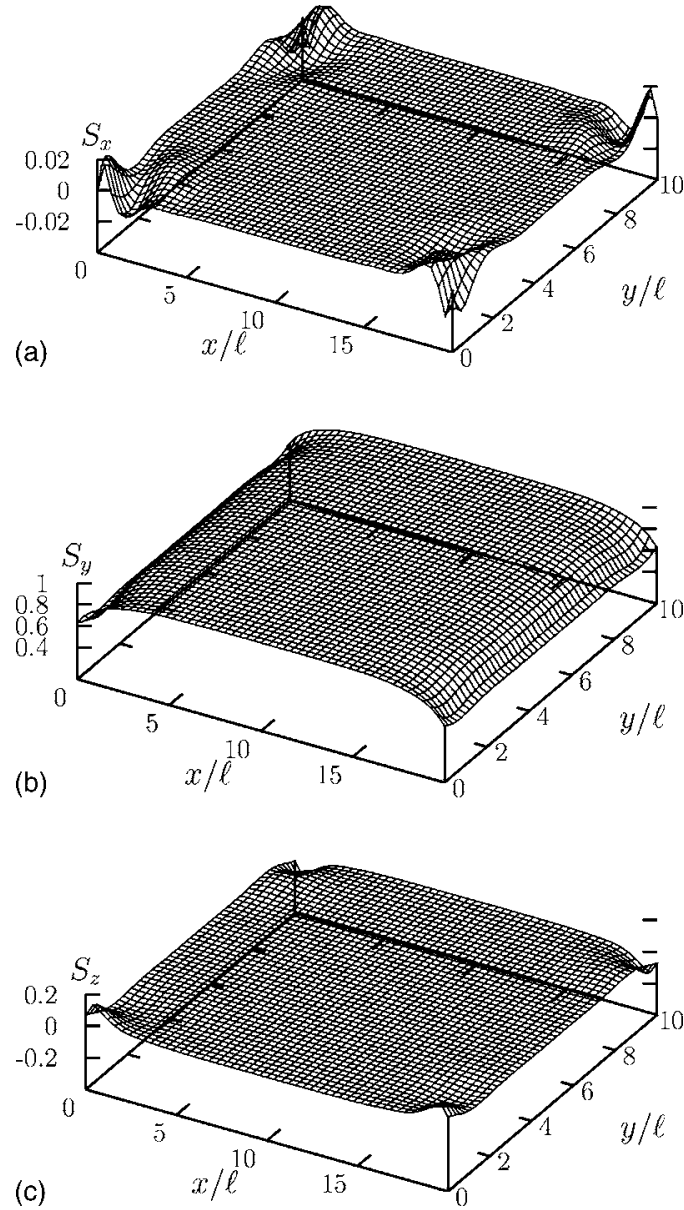

FIG. 2. Spin polarization in the presence of an electrical current flowing in the $x$ direction for a strip of length $L_{x}=20 l$ and $L_{y}=10 l$. The spin-orbit coupling strength is $\alpha=10^{-3} v_{F}$ and the elastic scattering rate is $1 / \tau=\alpha p_{F} / 2$. The spin polarization is given in units of the bulk value, $S_{0}=-|e| E \alpha \tau N_{0}$.

reached quickly. Typically we describe the Fermi surface with a set of 20 to $40 \hat{\mathbf{p}}_{i}$.

First we show numerical results for the spin polarization in the stationary limit. Figure 2 depicts the voltage induced spin polarization for $L_{x}=20 l, L_{y}=10 l, \alpha p_{F} \tau=2$, and $\alpha / v_{F}$ $=10^{-3}$; all our results are linear in the applied voltage, due to the linearity of the underlying equations. In the bulk, only the $S_{y}$ component is nonzero, and given by $S_{0}=-|e| E \alpha \tau N_{0}{ }^{2,13}$ A spin Hall effect induced spin polarization is found in the corners, as it is expected in Ref. 13. The spin polarization however is not purely in the $z$ direction but also has components in the $x$ direction.

Figure 3 shows $S_{y}\left(x, y=L_{y} / 2\right)$ by varying disorder. In the diffusive limit and assuming that the spin polarization vanishes at the interface to the leads, it has been predicted that ${ }^{13}$

$$
S_{y}(x)=S_{0}\left(1-\frac{\cosh \left[\left(x-L_{x} / 2\right) / L_{s}\right]}{\cosh \left(L_{x} / 2 L_{s}\right)}\right)
$$

where $L_{s}$ is the spin relaxation length. Apparently with the boundary condition we choose that a spin polarization still exists near $x=0, L_{x}$, in particular in the clean limit. Some

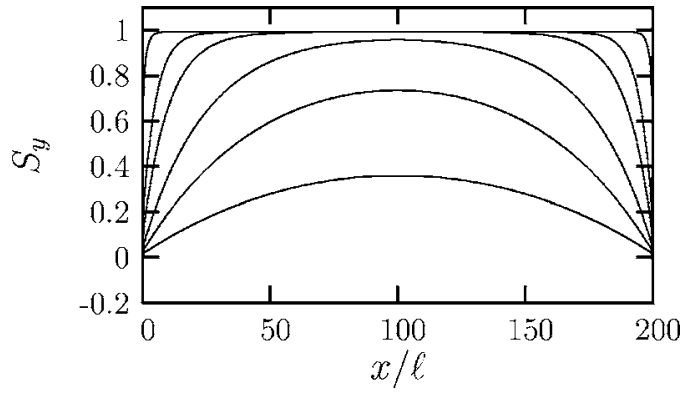

FIG. 3. $S_{y}$ in units of $S_{0}$ as a function of $x$ for $L_{x}=200 l, L_{y}$ $=100 l, \alpha / v_{F}=10^{-3}$, and $\alpha p_{F} \tau=0.005,0.01,0.02,0.05,0.1,1$ (from bottom to top).

mean free paths away from the interface, on the other hand, the data can be well fitted with an exponential increase or decrease, both in the clean and dirty limit. As a result we obtain the spin relaxation length as a function of disorder, shown in Fig. 4. In the dirty limit, $\alpha p_{F} \tau \ll 1$, our numerical result agrees with what is expected from the diffusion equation, $L_{s}=\sqrt{D \tau_{s}}=l / 2 \alpha p_{F} \tau$. In the clean limit the spin relaxation length is of the order of the mean free path, $L_{s}$ $\approx 1.27 l$, in agreement with what we find analytically, $\left(L_{s} / l\right)^{2}=(1+\sqrt{5}) / 2$.

In the following we will consider the time evolution of the spin polarization and the spin current. We start with a system in thermal equilibrium, switch on the voltage and observe the relaxation of the system into its stationary nonequilibrium state. It is a nontrivial problem to describe such a situation theoretically. One might be tempted to allow a timedependent voltage in the boundary condition, Eq. (39), and to follow then the time evolution. In this case the charge density becomes time dependent and inhomogeneous. This procedure makes sense for noninteracting electrons, but not for interacting electrons where the long range Coulomb interaction enforces charge neutrality. In principle, the interaction can be included into the quasiclassical formalism explicitly, see e.g., Ref. 34, however this is beyond the scope of the present paper.

Instead, we assume in the following that a voltage difference across the leads instantly results in a homogeneous electric field in the sample. Thus one must solve Eq. (13)



FIG. 4. Spin relaxation length $L_{s}$ in units of $l$ as a function of disorder, obtained by fitting the spatial dependence of the electric field induced spin polarization (shown in Fig. 3) using $S_{y}=a$ $+b\left\{\exp \left(-x / L_{s}\right)+\exp \left[-\left(L_{x}-x\right) / L_{S}\right]\right\}$. The diffusive limit expression is shown as a dashed line. 


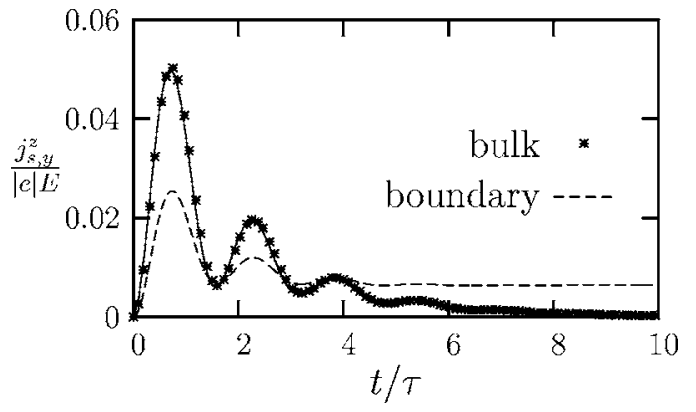

FIG. 5. Time evolution of the spin Hall current at the interface to the leads and in the bulk. In the bulk we compare our numerical result (data points) with the analytical result (full line) of Eq. (48). Near the leads, only numerical data are available (dashed curve). $j_{s, y}^{z}$ is evaluated at $y=L_{y} / 2, x=0$ (boundary) and $x=L_{x} / 2$ (bulk) for $L_{x}=20 l, L_{y}=10 l, \alpha / v_{F}=10^{-3}$ and $\alpha p_{F} \tau=2$.

with the initial condition $g(\mathbf{p}, \boldsymbol{\epsilon} ; \mathbf{x}, t)=\tanh (\epsilon / 2 T)\left(g^{R}-g^{A}\right)$ and taking into account the electric field via the substitution $\partial_{\mathbf{x}} \rightarrow \partial_{\mathbf{x}}-|e| \mathbf{E} \partial_{\boldsymbol{\epsilon}}$. In the numerics, however we find it more convenient to work in a scalar gauge, since then the (static) electric field disappears from the equation of motion and is present only in the initial condition and in the boundary condition. Generally the gauge transformation for the fields and the Green's function reads

$$
\begin{gathered}
\mathbf{A} \rightarrow \mathbf{A}+\partial_{\mathbf{x}} \chi \\
\phi \rightarrow \phi-\partial_{t} \chi \\
g_{t_{1} t_{2}} \rightarrow \exp \left\{-i|e|\left[\chi\left(t_{1}\right)-\chi\left(t_{2}\right)\right]\right\} g_{t_{1} t_{2}} .
\end{gathered}
$$

In the end we must solve Eq. (13) with the boundary condition (39) and the initial condition

$$
g^{K}(\hat{\mathbf{p}}, \boldsymbol{\epsilon} \mathbf{x}, t=0)=\tanh \left(\frac{\epsilon+|e| \phi(x)}{2 T}\right)\left(g^{R}-g^{A}\right),
$$

where $\phi(x)$ interpolates linearly between the two leads, $\phi(x)=V\left(L_{x} / 2-x\right) / L_{x}$.

In Fig. 5 we show the spin current $j_{s, y}^{z}$ as a function of time in the bulk and at the interface to the leads of a rather clean system $\left(\alpha p_{F} \tau=2, \alpha / v_{F}=10^{-3}\right)$. On short time scales the bulk current agrees with what we found ignoring disorder in Sec. IV: The spin current oscillates as a function of time with frequency $2 \alpha p_{F}$, the time average is given by the universal spin Hall conductivity. In the bulk, for the weakly disordered system we are considering, the time-dependent spin current is given by

$$
j_{s, y}^{z}=\frac{|e| E}{8 \pi}\left[\exp (-t / 2 \tau)-\exp (-3 t / 4 \tau) \cos \left(2 \alpha p_{F} t\right)\right]
$$

which can be obtained from the frequency dependent spin Hall conductivity given in the appendix. On the time scale of the spin relaxation time, here given by the scattering time $\tau$, the bulk spin current becomes exponentially suppressed and goes to zero in the stationary limit. Near the leads, on the other hand, the situation is somewhat different, since a finite spin current remains in the stationary limit.


FIG. 6. Spin Hall effect induced spin polarization $S_{z}$ in units of $S_{0}$ as a function of $y$ and $t$ at $x=L_{x} / 2$ for $L_{x}=20 l, L_{y}=10 l, \alpha / v_{F}$ $=10^{-3}$, and $\alpha p_{F} \tau=0.25,2,5$ (from bottom to top).

An important question is whether the spin current polarizes the electron system at the edges. In Fig. 6 we show the spin polarization in the $z$ direction across the system at $x$ $=L_{x} / 2$ as a function of time. Since in the early time evolution spin-current flows in the bulk, spin density accumulates near the edges. When the spin current disappears also the polarization vanishes. We see that the spin polarization at the edges oscillates as expected with frequency $2 \alpha p_{F}$. In the cleaner systems oscillations are of course faster. Remarkably, the maximum amplitude of oscillation relative to the bulk value is larger in the dirty system $\left(\alpha p_{F} \tau=0.25\right)$, where it is almost of the order of one.

This can be understood as follows: a rough estimate of the spin polarization at the edge is $S_{z} \sim \tau_{s} j_{s, y}^{z} / L_{s}$. With

$$
\begin{gathered}
\tau_{s} \sim \tau /\left(\alpha p_{F} \tau\right)^{2}, \\
j_{s, y}^{z} \sim e E\left(\alpha p_{F} \tau\right)^{2}, \\
L_{s} \sim l /\left(\alpha p_{F} \tau\right),
\end{gathered}
$$

the result is indeed $S_{z} \sim S_{0}=e E \alpha \tau N_{0}$. In the clean limit, on the other hand, the typical time and length scales are $\tau_{s} \sim \tau$ and $L_{s} \sim l$, from which we estimate $S_{z} \sim S_{0} /\left(\alpha p_{F} \tau\right)$, in agreement with our numerical findings. 


\section{SUMMARY}

We studied the spin Hall effect in a two-dimensional electron system by applying the method of quasiclassical Green's functions. The method has its strength in the description of macroscopic systems, i.e., systems with linear dimensions that are large compared to the Fermi wavelength. In particular we derived an Eilenberger-type equation in the presence of a generic spin-orbit coupling. We also showed that the method allows one to derive in an elegant way various results present in the literature. For the special case of the Rashba model we calculated numerically the spin Hall current and the spin Hall effect induced spin polarization on a strip. From our data we were able to extract quantitatively the spin relaxation length in the entire regime from the clean to the dirty limit, which is not covered by the diffusion equation approach. Although in the Rashba model the zerofrequency spin Hall conductivity is zero we found a spin Hall induced spin polarization at the edges on a short time scale after switching on the voltage. Our results were obtained using a boundary condition for the Green's function that corresponds to a smooth confining potential of the twodimensional electron gas. We expect the detailed structure of the spin polarization near the edges to depend on the boundary conditions, i.e., on the type of the confinement. Furthermore some caution is necessary concerning the spin polarization in the corners. The latter will depend both on the boundary condition and on the precise form of the distribution function near the opening to the leads. In our calculations we directly coupled the rectangular strip to reservoirs in thermal equilibrium. Near the corners this might lead to a relevant loss of information. In a more refined theory the distribution function at the opening to the leads will depend both on the transverse coordinate $y$ and on the properties of the leads. How boundary conditions and leads affect the spin polarization is certainly an experimentally relevant issue, which however is beyond the scope of this paper. We believe that our approach will stimulate further work in the field.

\section{ACKNOWLEDGMENTS}

The authors thank U. Eckern for valuable discussions. This work was supported by the Deutsche Forschungsgemeinschaft through Contract No. SFB 484.

\section{APPENDIX A: DIFFUSIVE LIMITS}

When spatial and temporal variations are slow it is in many cases convenient to study the equations of motion in the diffusive limit. Usually, in this limit the full angular dependent Green's function $\check{g}(\hat{\mathbf{p}})$ can be easily constructed from its angular average $\langle\check{g}(\hat{\mathbf{p}})\rangle$ so it is sufficient to study $\langle\check{g}(\hat{\mathbf{p}})\rangle$. To determine the equation for $\langle\check{g}(\hat{\mathbf{p}})\rangle$ we first write all the terms of Eq. (13) with $\stackrel{g}{ }$ on the left-hand side and those depending on $\langle\check{g}\rangle$ on the right-hand side. For the Keldysh component we hence get

$$
\left(M_{0}+M_{1}\right) g^{K}=\left(N_{0}+N_{1}\right)\left\langle g^{K}\right\rangle
$$

where

$$
\begin{gathered}
M_{0} g^{K}=g^{K}+\tau \partial_{t} g^{K}+v_{F} \hat{\boldsymbol{\tau}} \cdot \partial_{\mathbf{x}} g^{K}+i \tau\left[\mathbf{b}_{0} \cdot \boldsymbol{\sigma}, g^{K}\right], \\
M_{1} g^{K}=-\frac{1}{2} \tau\left\{\frac{\left(\mathbf{b}_{0} \cdot \boldsymbol{\sigma}\right) \hat{\mathbf{p}}}{p_{F}}-\partial_{\mathbf{p}}\left(\mathbf{b}_{0} \cdot \boldsymbol{\sigma}\right), \partial_{\mathbf{x}} g^{K}\right\} \\
-\frac{1}{2} i \tau\left[\partial_{\xi}\left(\mathbf{b}_{0} \cdot \boldsymbol{\sigma}\right),\left\{\mathbf{b}_{0} \cdot \boldsymbol{\sigma}, g^{K}\right\}\right]-\frac{1}{2}\left\{\left\langle\partial_{\xi} \mathbf{b}_{0} \cdot \boldsymbol{\sigma}\right\rangle, g^{K}\right\} \\
N_{0}\left\langle g^{K}\right\rangle=\left\langle g^{K}\right\rangle, \\
N_{1}\left\langle g^{K}\right\rangle=-\frac{1}{2}\left\{\partial_{\xi} \mathbf{b}_{0} \cdot \boldsymbol{\sigma},\left\langle g^{K}\right\rangle\right\}
\end{gathered}
$$

Here $M_{1}$ and $N_{1}$ are small in the expansion parameter $|\mathbf{b}| / \epsilon_{F}$. The Eilenberger equation is then rewritten as

$$
g^{K}=\left(M_{0}+M_{1}\right)^{-1}\left(N_{0}+N_{1}\right)\left\langle g^{K}\right\rangle,
$$

i.e., to first order in $|\mathbf{b}| / \epsilon_{F}$,

$$
g^{K}=\left(M_{0}^{-1}+M_{0}^{-1} N_{1}-M_{0}^{-1} M_{1} M_{0}^{-1}\right)\left\langle g^{K}\right\rangle,
$$

from which the equation for the $s$-wave component of the Green's function becomes

$$
\left(1-\left\langle M_{0}^{-1}\right\rangle-\left\langle M_{0}^{-1} N_{1}\right\rangle+\left\langle M_{0}^{-1} M_{1} M_{0}^{-1}\right\rangle\right)\left\langle g^{K}\right\rangle=0 .
$$

In the low frequency, long wavelength limit this is the generalized diffusion equation obtained in the literature in various limits. ${ }^{13,21,26}$ The explicit form is obtained by evaluating the angular average of the operator product $M^{-1} N$.

In the Rashba model for instance where $\mathbf{b}=\alpha \mathbf{p} \times \hat{\mathbf{e}}_{z}$ one finds

$$
\begin{gathered}
M_{0}=\left(\begin{array}{cccc}
L & 0 & 0 & 0 \\
0 & L & 0 & a \hat{p}_{x} \\
0 & 0 & L & a \hat{p}_{y} \\
0 & -a \hat{p}_{x} & -a \hat{p}_{y} & L
\end{array}\right), \\
M_{1}=\left(\begin{array}{cccc}
0 & Q_{y} & -Q_{x} & 0 \\
Q_{y} & 0 & 0 & 0 \\
-Q_{x} & 0 & 0 & 0 \\
0 & 0 & 0 & 0
\end{array}\right)
\end{gathered}
$$

with

$$
\begin{gathered}
L=1+\tau \partial_{t}+v_{F} \tau \hat{\mathbf{p}} \cdot \partial_{\mathbf{x}}, \\
a=2 \alpha p_{F} \tau, \\
Q_{x, y}=\alpha \tau\left[\partial_{x, y}-\left(\hat{\mathbf{p}} \cdot \partial_{\mathbf{x}}\right) \hat{p}_{x, y}\right]
\end{gathered}
$$

and

$$
N_{0}+N_{1}=\left(\begin{array}{cccc}
1 & -\alpha \hat{p}_{y} / v_{F} & \alpha \hat{p}_{x} / v_{F} & 0 \\
-\alpha \hat{p}_{y} / v_{F} & 1 & 0 & 0 \\
\alpha \hat{p}_{x} / v_{F} & 0 & 1 & 0 \\
0 & 0 & 0 & 1
\end{array}\right) .
$$

In a dirty system, where $a \ll 1$, the result reads 


$$
\begin{gathered}
\left(\begin{array}{cccc}
\partial_{t}-D \partial_{\mathbf{x}}^{2} & -2 B \partial_{y} & 2 B \partial_{x} & 0 \\
-2 B \partial_{y} & \partial_{t}-D \partial_{\mathbf{x}}^{2}+\tau_{s}^{-1} & 0 & -2 C \partial_{x} \\
2 B \partial_{x} & 0 & \partial_{t}-D \partial_{\mathbf{x}}^{2}+\tau_{s}^{-1} & -2 C \partial_{y} \\
0 & 2 C \partial_{x} & 2 C \partial_{y} & \partial_{t}-D \partial_{\mathbf{x}}^{2}+2 \tau_{s}^{-1}
\end{array}\right) \\
\quad\left(\begin{array}{c}
\left\langle g_{0}^{K}\right\rangle \\
\left\langle g_{x}^{K}\right\rangle \\
\left\langle g_{y}^{K}\right\rangle \\
\left\langle g_{z}^{K}\right\rangle
\end{array}\right)=0,
\end{gathered}
$$

where $D=\frac{1}{2} v_{F}^{2} \tau$ is the diffusion constant and

$$
B=\frac{\alpha a^{2}}{1+a^{2}}, \quad C=\frac{v_{F} a}{2\left(1+a^{2}\right)^{2}}, \quad \frac{1}{\tau_{s}}=\frac{1}{2 \tau} \frac{a^{2}}{1+a^{2}} .
$$

In the clean limit $(a \gtrsim 1)$ there is no spin diffusion, and Eq. (A15) is not justified. However the equation is constructed in such a way that it applies in the clean limit for a space- and time-independent spin polarization.

In the general case (arbitrary $a$ ) we investigate spin relaxation for a spatially homogeneous system, where the equation of motion reads

$$
\begin{gathered}
{\left[L\left(L^{2}+a^{2}\right)-L^{2}-\frac{1}{2} a^{2}\right]\left\langle g_{x, y}^{K}\right\rangle=0,} \\
{\left[L\left(L^{2}+a^{2}\right)-L^{2}\right]\left\langle g_{z}^{K}\right\rangle=0,}
\end{gathered}
$$

with $L=1+\tau \partial_{t}$. Clearly the spin dynamics for each component is determined from three relaxation times. For the $z$ component, e.g., these are

$$
\begin{gathered}
\frac{1}{\tau_{1}}=\frac{1}{\tau}, \\
\frac{1}{\tau_{2,3}}=\frac{1}{2 \tau} \pm \frac{1}{2 \tau} \sqrt{1-4 a^{2}} .
\end{gathered}
$$

For dirty systems the longest of these times is $\tau_{3} \approx \tau / a^{2}$, in agreement with what we find from the diffusion equation (A15). In the clean limit the "relaxation" time becomes complex corresponding to an oscillating spin polarization.

\section{APPENDIX B: SPIN HALL CONDUCTIVITY}

We go back to (A1) and solve it for an infinite system under the influence of a uniform but time-dependent electric field in $x$ direction. We choose the vector gauge as in Sec. IV, i.e., $\partial_{\mathbf{x}} \rightarrow \partial_{\mathbf{x}}-|e| E \partial_{\epsilon}$. To linear order in the external field and transforming to Fourier space, (A1) becomes

$$
M_{0} g^{K}=\left(1+N_{1}\right)\left\langle g^{K}\right\rangle+S_{E},
$$

where $M_{0}$ and $N_{1}$ are the same as before, $S_{E}$ is a source term due to the electric field,

$$
S_{E}=\widetilde{E}\left[\left(\begin{array}{c}
\hat{p}_{x} \\
0 \\
0 \\
0
\end{array}\right)+\frac{\alpha}{v_{F}}\left(\begin{array}{c}
0 \\
-2 \hat{p}_{x} \hat{p}_{y} \\
\hat{p}_{x}^{2}-\hat{p}_{y}^{2} \\
0
\end{array}\right)\right]
$$

with $\widetilde{E}=|e| l E(\omega) \partial_{\epsilon} g_{0}^{K \text {,eq }}$. Inverting $M_{0}$ and performing the angular average one obtains

$$
\left\langle\hat{p}_{y} g_{z}^{K}\right\rangle=-\frac{a}{2\left(L^{2}+a^{2}\right)}\left(\frac{\alpha}{v_{F}} \tilde{E}-\left\langle g_{y}^{K}\right\rangle\right)
$$

where the two terms in the large parentheses correspond to bubble and vertex corrections, respectively, in the diagrammatic language. Furthermore we see that the spin polarization along $\mathbf{e}_{y}$ contributes to the spin Hall current. The spin polarization along $\mathbf{e}_{y}$ is obtained by inverting $M_{0}$ and performing the angular average

$$
\left\langle g_{y}^{K}\right\rangle=\frac{\alpha}{v_{F}} \frac{a^{2} \widetilde{E}}{2\left[L\left(L^{2}+a^{2}\right)-\left(L^{2}+\frac{a^{2}}{2}\right)\right]} .
$$

In the static limit $(L=1)$ this reads $\left\langle g_{y}^{K}\right\rangle=\frac{\alpha}{v_{F}} \widetilde{E}$, corresponding to the bulk value $S_{0}$, quoted in the text. This value for $\left\langle g_{y}^{K}\right\rangle$ leads to the cancellation discovered previously ${ }^{35}$ and to the vanishing of the static spin Hall conductivity. Finally, by combining Eqs. (B3) and (B4), one obtains the frequency dependent spin Hall current with polarization along $\mathbf{e}_{z}$ as

$$
j_{s, y}^{z}(\omega)=\sigma_{s H}(\omega) E(\omega)=\frac{|e|}{8 \pi} \frac{-i \omega \tau a^{2}}{L\left(L^{2}+a^{2}\right)-\left(L^{2}+\frac{a^{2}}{2}\right)} E(\omega)
$$

which agrees with the result given by both Mishchenko et al. ${ }^{13}$ and Chalaev and Loss. ${ }^{17}$ To examine the transient response in time, we use $E(t)=E \theta(t)$ so that

$$
j_{s, y}^{z}(t)=E \int_{-\infty}^{t} d t^{\prime} \sigma_{s H}\left(t^{\prime}\right)
$$

where

$$
\sigma_{s H}(t)=\int_{-\infty}^{\infty} \frac{d \omega}{2 \pi} \sigma_{s H}(\omega) e^{-i \omega t}
$$

The poles of the integrand may be found by expressing $\omega \tau$ $=x+i y$ and setting to zero the real and imaginary part of the cubic polynomial in the denominator of $\sigma_{s H}(\omega)$,

$$
\begin{gathered}
x\left[x^{2}-3 y^{2}-4 y-\left(1+a^{2}\right)\right]=0, \\
x^{2}(3 y+2)-y^{3}-2 y^{2}-y\left(1+a^{2}\right)-\frac{a^{2}}{2}=0 .
\end{gathered}
$$


In the large $a$ limit, one obtains the three solutions

$$
x=0, \quad y=-1 / 2 ; \quad x= \pm a, \quad y=-3 / 4 .
$$

Computing the residues with the same accuracy yields

$$
\begin{aligned}
\sigma_{s H}(t)= & -\frac{|e|}{8 \pi} \frac{\theta(t)}{2 \tau}\left[e^{-t / 2 \tau}-e^{-3 t / 4 \tau} \cos (a t / \tau)\right. \\
& \left.-2 a e^{-3 t / 4 \tau} \sin (a t / \tau)\right] .
\end{aligned}
$$

Finally, inserting the above result into Eq. (B6), one obtains - to leading order in $1 / a$ - the time-dependent current quoted in the text.
${ }^{1}$ M. I. Dyakonov and V. I. Perel, Phys. Lett. 35A, 459 (1971).

${ }^{2}$ V. M. Edelstein, Solid State Commun. 73, 233 (1990); J. Phys.: Condens. Matter 5, 2603 (1993).

${ }^{3}$ J. E. Hirsch, Phys. Rev. Lett. 83, 1834 (1999).

${ }^{4}$ S. Murakami, N. Nagaosa, and S.-C. Zhang, Science 301, 1348 (2003).

${ }^{5}$ J. Sinova, D. Culcer, Q. Niu, N. A. Sinitsyn, T. Jungwirth, and A. H. MacDonald, Phys. Rev. Lett. 92, 126603 (2004).

${ }^{6}$ Y. K. Kato, R. C. Myers, A. C. Gossard, and D. D. Awschalom, Science 306, 1910 (2004).

${ }^{7}$ J. Wunderlich, B. Kaestner, J. Sinova, and T. Jungwirth, Phys. Rev. Lett. 94, 047204 (2005).

${ }^{8}$ N. A. Sinitsyn, E. M. Hankiewicz, W. Teizer, and J. Sinova, Phys. Rev. B 70, 081312(R) (2004).

${ }^{9}$ S.-Q. Shen, Phys. Rev. B 70, 081311(R) (2004).

${ }^{10}$ M.-C. Chang, Phys. Rev. B 71, 085315 (2005).

${ }^{11}$ A. V. Shytov, E. G. Mishchenko, H.-A. Engel, and B. I. Halperin, Phys. Rev. B 73, 075316 (2006).

${ }^{12}$ J. I. Inoue, G. E. W. Bauer, and L. W. Molenkamp, Phys. Rev. B 70, 041303(R) (2004).

${ }^{13}$ E. G. Mishchenko, A. V. Shytov, and B. I. Halperin, Phys. Rev. Lett. 93, 226602 (2004).

${ }^{14}$ A. Khaetskii, Phys. Rev. Lett. 96, 056602 (2006).

${ }^{15}$ R. Raimondi and P. Schwab, Phys. Rev. B 71, 033311 (2005).

${ }^{16}$ O. V. Dimitrova, Phys. Rev. B 71, 245327 (2005).

${ }^{17}$ O. Chalaev and D. Loss, Phys. Rev. B 71, 245318 (2005).
${ }^{18}$ K. Nomura, J. Sinova, N. A. Sinitsyn, and A. H. MacDonald, Phys. Rev. B 72, 165316 (2005).

${ }^{19}$ E. I. Rashba, Phys. Rev. B 70, 201309(R) (2004).

${ }^{20}$ S. Murakami, Phys. Rev. B 69, 241202(R) (2004).

${ }^{21}$ A. G. Mal'shukov, L. Y. Wang, C. S. Chu, and K. A. Chao, Phys. Rev. Lett. 95, 146601 (2005).

${ }^{22}$ A. Khaetskii, Phys. Rev. B 73, 115323 (2006).

${ }^{23}$ S. Y. Liu and X. L. Lei, Phys. Rev. B 72, 155314 (2005).

${ }^{24}$ B. K. Nikolic, S. Souma, L. P. Zarbo, and J. Sinova, Phys. Rev. Lett. 95, 046601 (2005).

${ }^{25}$ M. Onoda and N. Nagaosa, Phys. Rev. B 72, 081301(R) (2005).

${ }^{26}$ A. A. Burkov, A. S. Nunez, and A. H. MacDonald, Phys. Rev. B 70, 155308 (2004).

${ }^{27}$ J. Rammer and H. Smith, Rev. Mod. Phys. 58, 323 (1986).

${ }^{28}$ A. V. Zaitsev, Zh. Eksp. Teor. Fiz. 86, 1742 (1984). [Sov. Phys. JETP 59, 1015 (1984)].

${ }^{29}$ A. L. Shelankov, J. Low Temp. Phys. 60, 29 (1985).

${ }^{30}$ A. Millis, D. Rainer, and J. A. Sauls, Phys. Rev. B 38, 4504 (1988).

${ }^{31}$ I. Adagideli and G. E. W. Bauer, Phys. Rev. Lett. 95, 256602 (2005).

${ }^{32}$ V. M. Galitski, A. A. Burkov, and S. Das Sarma, cond-mat/ 0601677 (unpublished).

${ }^{33}$ P. G. Silvestrov and E. G. Mishchenko, cond-mat/0506516 (unpublished).

${ }^{34}$ P. Schwab and R. Raimondi, Ann. Phys. 12, 471 (2003).

${ }^{35}$ P. Schwab and R. Raimondi, Eur. Phys. J. B 25, 483 (2002). 